\title{
Effects of Polymer Infusion and Characteristic Length Scale on Gold-Black Long-Wave and Far-Infrared Absorbance
}

\author{
Justin W. Cleary ${ }^{1}$, Robert E. Peale ${ }^{1}$, Masahiro Ishigami ${ }^{1}$, Christian W. Smith ${ }^{1}$, Kevin Baillie ${ }^{1}$, Josh E. Colwell ${ }^{1}$, \\ Oliver Edwards ${ }^{2}$ and Chris J. Fredricksen ${ }^{3}$ \\ 1. Department of Physics, University of Central Florida, Orlando FL, 32816, United States \\ 2. Zyberwear, Inc., Ocoee FL, 34761, United States \\ 3. LRC Engineering, Inc., Orlando FL, 32825, United States
}

Received: July 28, 2010 / Accepted: August 23, 2010 / Published: February 10, 2011.

\begin{abstract}
Infrared absorbance is investigated in gold-black, a porous nano-structured conducting film. A two level full factorial optimization study with evaporation-chamber pressure, boat current, substrate temperature, and degree of polymer infusion (for hardening) was performed. Polymer infusion was found generally to reduce absorbance in the long wave IR but has little effect at THz wavelengths, although for samples with the highest absorbance there is a slight improvement in the absorbance figure of merit (FOM) in both wavelength regimes. The characteristic length scales of the structured films vary considerably as a function of deposition parameters, but the IR FOM is found to be weakly correlated with these distributions, which are determined by wavelet analysis of scanning electron micrographs.
\end{abstract}

Key words: Gold-black, infrared absorber, full-factorial, polymer hardening, wavelet analysis.

\section{Introduction}

Metal-black is a nano-structured conducting film that has been exploited as a broad-band surface absorber for bolometers [1-3]. This paper considers the effect of hardening a gold-black film via polymer infusion and the potential correlation of IR absorption to the characteristic length scales of the films. We have employed a full factorial approach whereby factors affecting the optical properties of the films are varied all together, as opposed to one-at-a-time experiments where one factor is varied while the others are held constant. Responses were measured for the film produced from each experiment and statistically significant interaction terms were calculated. Response

Corresponding author: Robert E. Peale (1961- ), male, tenured professor, research field: Far-IR/Terahertz spectroscopy and technology development. E-mail: peale@ mail.ucf.edu. surfaces were generated in order to find maxima and minima within the factor space. Additionally, wavelet analysis was employed to establish a relationship between characteristic morphological length scales of the films and a figure of merit related to the optical absorbance. We emphasize the long wave infrared and the underutilized far-IR, or THz, spectral regions.

Metal blacks have been investigated for their optical properties since the 1930's [4]. Pfund deposited metal-blacks on nitrocellulose films in an environment of nitrogen or air. Harris studied gold-black films from the late 1940's into the 1960's [5-9]. The films were prepared similarly to Pfund's (deposited on thin membranes of cellulose nitrate in a partial atmosphere of nitrogen), with emphasis on the pressure of nitrogen and the evaporation rate as prime factors determining the morphology of the films. Optical properties were 
studied by Harris from the wavelength range of 3 to 15 microns [5], and later from 15 to 110 microns [8]. Deposition by evaporation in an inert gas at tenths to tens of Torr has followed the method of Harris with little change. Attempts to link particle size and packing fraction to pressure at He pressures between 1 and 20 Torr [1, 2] have been carried out using this methodology. Investigation of gold-black films containing small percentages of copper [10] revealed that loading samples in the chamber in a high humidity environment lead to a decrease in absorbance. Becker, Fettig, and Ruppel [11] studied the electrical conductivity of gold-black films extensively in order to correlate the electrical properties with the optical properties.

One objective of this work was to develop a mechanically stable absorber for an innovative MEMS-based bolometric infrared imaging array detector with high frame rates. Metal blacks are produced in a low-vacuum process, resulting in a porous nano-structured metallic film having a broad particle-size distribution. The morphology depends on the pressure of the inert gas in the evaporation chamber, the deposition rate, and the temperature of the substrate [5]. A correlation between the absorptivity of gold black and the resistivity of the thin films has been exploited [11] to verify the expected properties of as-grown films. Here the possibility of a correlation between absorption and the particle size distribution is investigated.

This paper presents a number of approaches that are innovative. It appears to be the first use of wavelet analysis on SEM (scanning electron microscope) images of gold black in order to quantify particle length-scale distributions. It appears to be the first attempt to harden the usually fragile gold-black films by polymer infusion. It appears also to be the first use of the structured statistical approach to the design of experiments, known as the two-level full factorial method, for optimizing the absorbance of gold-black films.

\section{Experiment}

Gold-black films were produced by thermal evaporation in an inert gas, either nitrogen or helium. Double-side polished silicon substrates, two per deposition for different characterization techniques, were mounted on a copper plate attached to a thermoelectric cooler (TEC), which is attached to a water-cooled copper heat sink, allowing temperature control of the substrate. Molybdenum boats with a trough of dimensions $12 \mathrm{~mm} \times 5 \mathrm{~mm} \times 1 \mathrm{~mm}$ deep were filled with a $1.5 \mathrm{~cm}$ length of $0.813 \mathrm{~mm}$ diameter 99.9\% pure gold wire for each deposition. The chamber is pumped to below $10^{-4}$ Torr, at which point 1Torr of inert gas is introduced. The deposition is then completed with an evaporation current specific to each experiment. Deposition continued until the boat was visibly empty. The films are sufficiently thin, approximately $50 \mathrm{~nm}$, to allow some transmission of an optical beam.

Polymer infusion by cyanoacrylate fuming on the deposited gold-black samples with a process similar to that described in Ref. [12]. A standard cup warmer was allowed to reach maximum temperature inside an enclosed fume chamber. The sample was mounted above the cup warmer at a height of 4 inches. Small aluminum foil boats with $0.69 \mathrm{~mL}$ of cyanoacrylate were placed on the heated warmer to begin the fuming process.

Initial screening experiments determined the parameters of interest and their useful ranges, resulting in samples designated series "A" and series "B" [13]. Then, a two level full factorial optimization scheme [14], including polymer infusion, was followed to determine the conditions that produce maximum absorbance, giving samples in a series labeled " $\mathrm{C}$ ". The protocol is the most statistically sound and economical approach to optimization when underlying physics is poorly understood [14]. Parameters chosen to be varied were He pressure, evaporation current, and substrate temperature. Each parameter took one of two values. In addition, after characterizing these samples, the polymer infusion was completed with two different 
fuming times. With 4 parameters, there are $2^{4}$ or 16 different combinations. Four intermediate points were also included to reveal curvature. A matrix of experiments (Table 1) was set up so that every combination of parameter values in the scheme is completed in random order to avoid systematic errors. The location in parameter space that optimized the films (as characterized by a figure of merit to be defined) and correlations between parameters are then discovered.

Table 1 gives all characterized Au-black samples with corresponding experimental conditions. The low and high values for $\mathrm{P}$ are 1 and 2 Torr, for $\mathrm{T}$ are $40^{\circ}$ and $60^{\circ} \mathrm{F}$, for I are 52 and 56 amps, and for polymer infusion are 1 and $2 \mathrm{~h}$. Sample C02 was destroyed before it could be measured. Its conditions were duplicated in another sample.

Infrared absorption was determined from transmittance and reflectance spectra collected using a BOMEM DA8 Fourier transform spectrometer. Resources were

Table 1 Matrix of experiments in two-level full factorial optimization of gold black. High (+), low (-), and intermediate $(*)$ levels are indicated. Bold samples are those with the best FOM.

\begin{tabular}{lllll}
\hline Sample & P & T & I & Polymer \\
\hline C01 & + & + & - & + \\
C03 & + & - & - & - \\
C04 & + & - & - & + \\
C05 & $*$ & $*$ & $*$ & $*$ \\
C06 & - & - & - & - \\
C07 & - & + & - & + \\
C08 & + & + & - & - \\
C09 & $*$ & $*$ & $*$ & $*$ \\
C10 & + & - & + & + \\
C11 & + & + & + & + \\
C12 & - & + & + & - \\
C13 & - & + & + & + \\
C14 & + & - & + & - \\
C15 & - & - & - & + \\
C16 & - & - & + & + \\
C17 & - & - & + & - \\
C18 & - & + & - & - \\
C19 & + & + & + & - \\
C20 & $*$ & $*$ & $*$ & $*$ \\
C21 & $*$ & $*$ & $*$ & $*$ \\
\hline
\end{tabular}

a globar or mercury lamp source, a $4 \mathrm{~K}$ Bolometer, HgCdTe or DTGS detector, and Mylar or $\mathrm{KBr}$ beamsplitters. Transmittance measurements at normal incidence used a clean double side polished silicon substrate as a reference. Specular reflectance at $30 \mathrm{deg}$. angle of incidence was referenced to a gold-coated silicon substrate. The figure of merit (FOM) was defined as 1-T-R, a measure of absorbance. It is not exactly the same as absorbance, however, since the reflectance was not at normal incidence. To determine the effect of the polymer infusion on IR absorbance, the spectra where measured on the 1st set of 20 gold-black samples pre- and post-polymer infusion.

SEM images were collected on the 2nd set of 20 gold-black samples, which did not undergo polymer infusion. A discrete wavelet analysis was performed on each image using a Morlet wavelet [15, 16]. The wavelet transform was calculated for each row of each image. The wavelet transform provides a map of the power present at all wavelengths at different regions of the image, or in this case at different locations within each image row. The wavelet power was integrated along the image row and power for all rows was summed since there is interest in the overall wavelength power distribution, not spatial variations within the images. The amplitude of the wavelet spectrum is in arbitrary units.

\section{Results}

\subsection{Effects of Pressure, Temperature and Current}

Infrared FOM was determined at 150 and $600 \mathrm{~cm}^{-1}$ wavenumbers, corresponding roughly to the $\mathrm{THz}$ and long wave IR (LWIR) bands. The results are summarized in Fig. 1. The samples are numbered as presented in Table 1. The FOM values at 150 and 600 $\mathrm{cm}^{-1}$ are strongly correlated. The samples with highest FOM are indicated in bold in Table 1. From these data, a full factorial process was completed on all samples before polymer infusion. From these full factorial results using FOM as a response factor, the evaporation current is found to be the most significant factor followed 


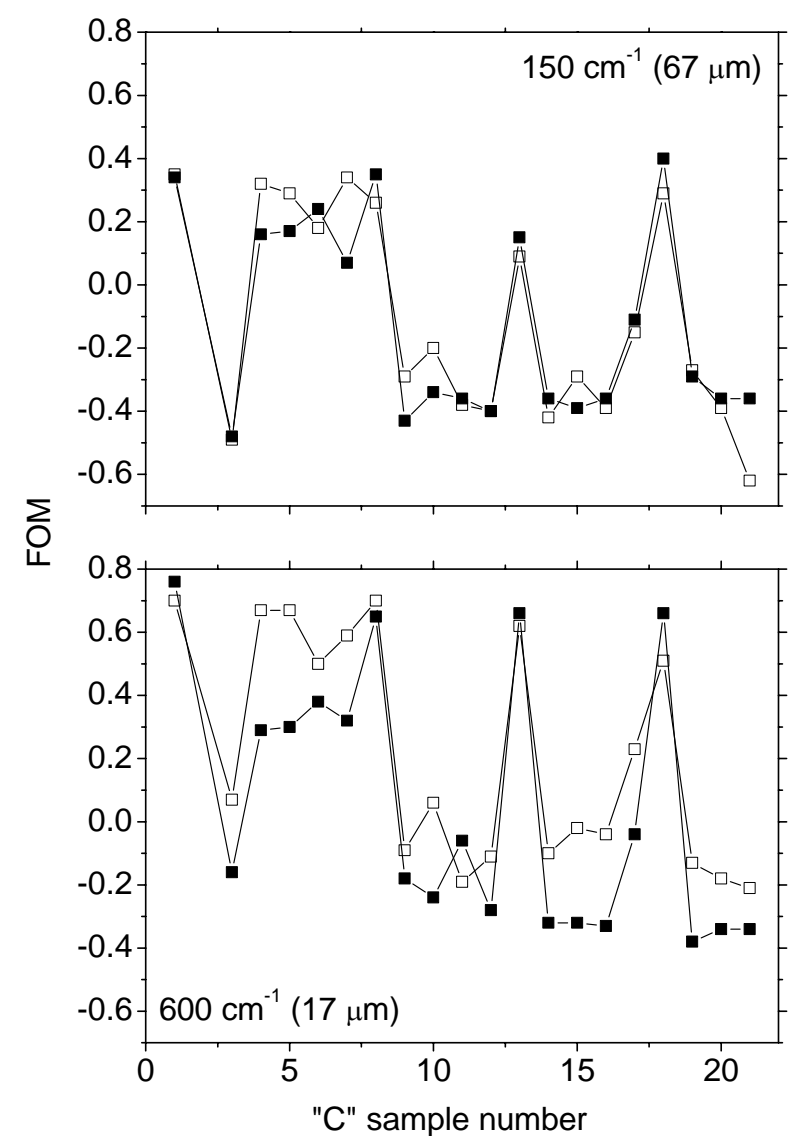

Fig. 1 Figures of merit at THz (above) and long-wave IR (below) frequencies for gold-black samples of Table 1. Solid (open) symbols indicate values after (before) polymer infusion.

by substrate temperature and the temperature-current interaction at $150 \mathrm{~cm}^{-1}$. At $600 \mathrm{~cm}^{-1}$, these factors are similarly significant while the pressure-current interaction also becomes significant. The results indicate the lower current setting, or hence lower deposition rate, gives higher absorbance at both wavelengths. The initial substrate temperature appears to have a moderate effect on the absorbance, but it was determined that the small window between high and low settings give inconclusive results on this effect. The high and low pressure settings used here appear to have little effect on the absorbance. The full-factorial analysis shows some curvature, although this appears to be insignificant.

\subsection{Effect of Polymer Infusion}

Fig. 1 reveals a tendency for polymer infusion to
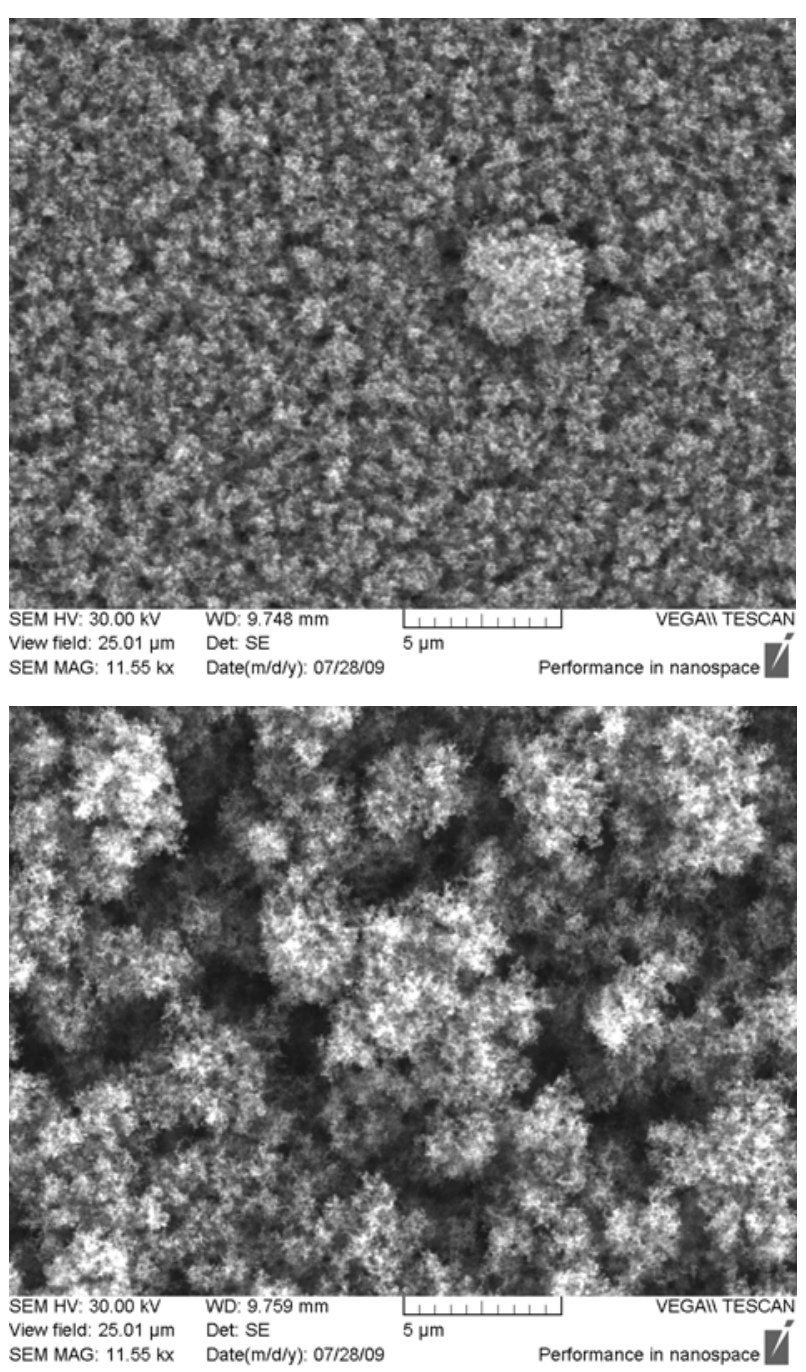

Fig. 2 SEM images of sample C04 (above) and C09 (below). Both images have 25 micron field of view.

reduce the FOM at $600 \mathrm{~cm}^{-1}$. However, this is less significant at $150 \mathrm{~cm}^{-1}$. For the samples with highest FOM there is a tendency for polymer infusion to increase the figure of merit slightly.

\subsection{Effect of Particle Length-Scale Distribution}

Fig. 2 presents SEM images of two of the gold-black samples from Table 1 . The metallic particles are arranged in interconnected groups with a broad-range of characteristic length scales or spatial wavelengths. These two images were chosen for presentation because of the large difference in their characteristic length scales.

Fig. 3 presents the wavelet analysis of SEM images 


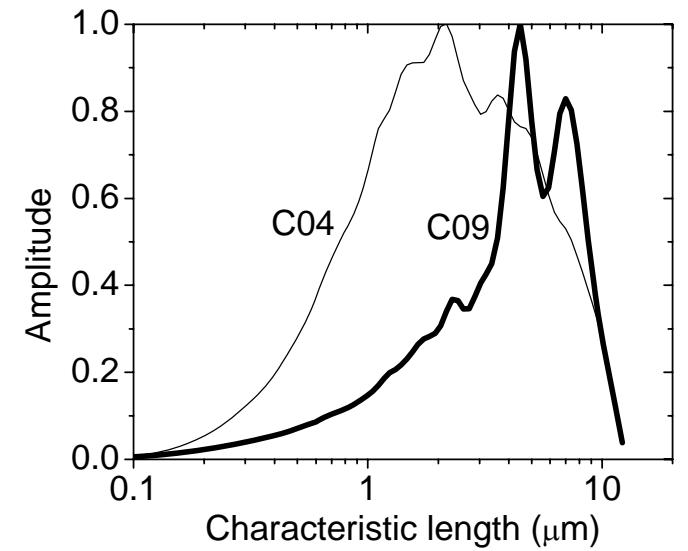

Fig. 3 Wavelet analysis of SEM images for samples C04 and C09. The images had 50 micron field of view. The amplitude scale is normalized.

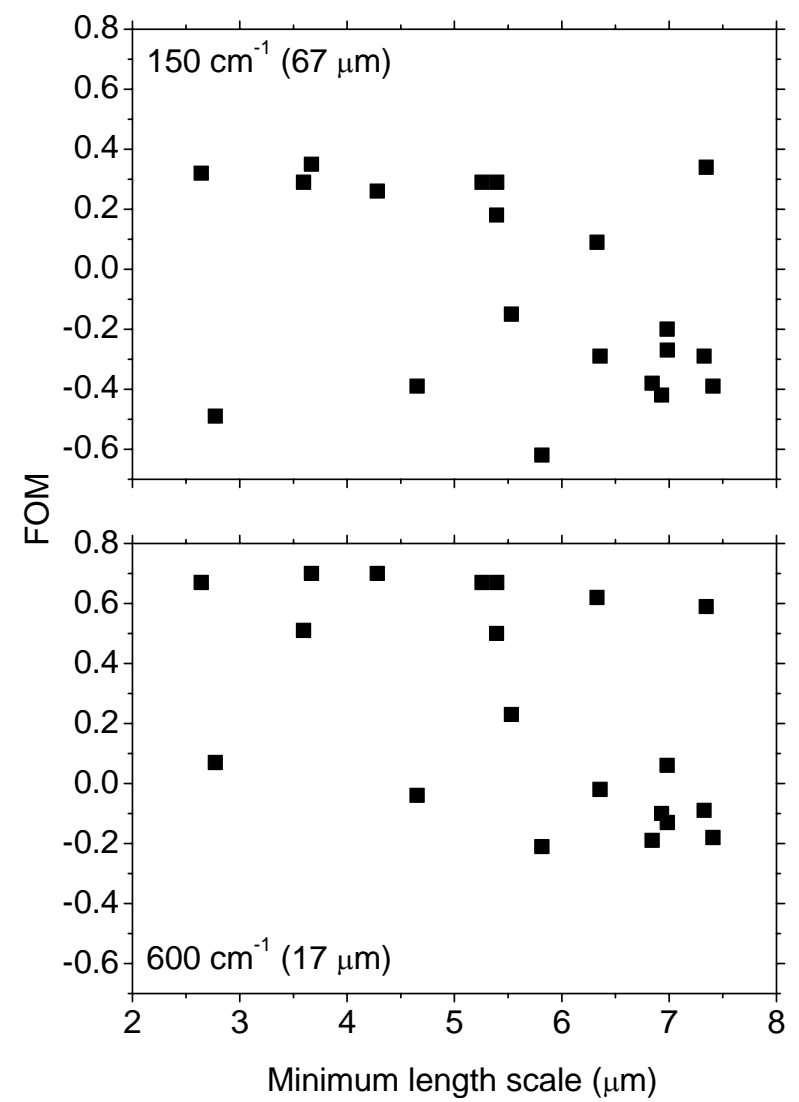

Fig. 4 Figure of merit at THz (above) and long-wave IR (below) frequencies as function of minimum characteristic length scale for gold-black.

for C04 and C09 with 50 micron field of view. The wavelet power spectrum is characterized by a series of over lapping peaks. The upper wavelength cutoff is determined by the maximum dimension of the image. For two images with the same magnification, the lower limit is a quantitative measure of the minimum characteristic length scale in the image. We define the minimum characteristic length scale as the point where the power is half the maximum value.

Fig. 4 plots FOM values at $\mathrm{THz}$ and LWIR wavelengths as a function of the minimum length scale from the wavelet analysis of images with 50 micron FOV. At each wavelength, the FOM values appear in roughly two groups. Within each group there is no clear correlation between FOM and length scale. However considering both groups simultaneously, there appears to be a slight tendency for the high FOM points to be clustered at low length scale and vice versa. In other words, there may be a tendency for samples with smaller length scales to give higher absorbance.

The full factorial approach is now applied using the minimum characteristic length scale as a response factor (pre polymer infusion as before). The most significant factor is found again to be the evaporation current with the current-pressure interaction also playing a large role. The next largest factor is the interaction effect between the three deposition parameters. This full factorial indicates that lower evaporation current could lead to small length scales, which may also indicate higher absorbance as Fig. 4 shows.

\section{Conclusions}

The gold black film absorbance at long-wave IR and far-IR wavelengths is improved by slow depositions, or smaller evaporation current. The initial substrate temperatures may have an effect on IR absorbance, but the limited window between settings makes this result inconclusive. Weak dependence was found on the pressure within the range where these gold-black films are formed.

Hardening of gold-black by polymer infusion lowers the LWIR absorbance somewhat, but there is little effect in the THz spectral range. This suggests that the hardening process is particularly well suited for far-IR detector coatings. 
The characteristic minimum length scale of gold-black clusters in the structured films varies considerably as a function of deposition parameters. A smaller minimum length scale of gold-black clusters appears to indicate a higher possibility for higher IR absorbance. As with the absorbance measurements, smaller length scales may be achieved by slower deposition.

\section{Acknowledgments}

REP and JWC are supported by AFOSR under grant FA95501010030. JEC and KB were supported by NASAs Cassini Data Analysis Program Grant NNX08AJ68G. The contributions of LRC Engineering were supported by an AFOSR Phase I STTR FA9550-10-C-0069.

\section{References}

[1] C. Doland, P. O’Neill, A. Ignatiev, Particulate nature of solar absorbing films: Gold black, J. Vac. Sci. Tech. 14 (1977) 259-262.

[2] P. O’Neill, C. Doland, A. Ignatiev, Structural composition and optical properties of solar blacks: Gold black, Appl. Opt. 16 (1977) 2822-2826.

[3] N. Nelms, J. Dowson, Goldblack coating for thermal infrared detectors, Sens. Actuators A. 120 (2005) 403-407.

[4] A.H. Pfund, The optical properties of metallic and crystalline powders, J. Opt. Soc. Am. 23 (1933) 375-378.

[5] L. Harris, R.T. McGinnies, B.M. Siegel, The preparation and optical properties of gold blacks, J. Opt. Soc. Am. 38 (1948) 582-589.
[6] L. Harris, J.K. Beasley, The infrared properties of gold smoke deposits, J. Opt. Soc. Am. 42 (1952) 134-140.

[7] L. Harris, A.L. Loeb, Conductance and relaxation time of electrons in gold blacks from transmission and reflection measurements in the far infrared, J. Opt. Soc. Am. 43 (1953) 1114-1118.

[8] L. Harris, Reflectance of goldblack deposits, and some other materials of low reflectance from 254 microns to 1100 millimicrons. The scattering-unit size in goldblack deposits, J. Opt. Soc. Am. 46 (1956) 160-163.

[9] L. Harris, The transmittance and reflectance of gold black deposits in the 15- to 100-micron region, J. Opt. Soc. Am. 51 (1961) 80-82.

[10] D. Advena, V. Bly, J. Cox, Deposition and characterization of far-infrared absorbing gold black films, Appl. Opt. 32 (1993) 1136-1144.

[11] W. Becker, R. Fettig, W. Ruppel, Optical and electrical properties of black gold layers in the far infrared, Infrared Phys. and Tech. 40 (1999) 431-445.

[12] P. Czekanski, M. Fasola, J. Allison, A mechanistic model for the superglue fuming of latent fingerprints, J. Forensic Sci. 51 (2006) 1323-1328.

[13] J.W. Cleary, Surface plasmon hosts for infrared waveguides and biosensors, and plasmons in gold-black nano-structured films, Doctoral dissertation, University of Central Florida, Orlando, FL, 2010

[14] S. Wolf, R.N. Tauber, Silicon Processing for the VLSI Era, Vol. 1-Process Technology, Lattice, Ch. 18, Sunset Beach CA, 1986.

[15] G. Foster, Wavelets for period analysis of unevenly sampled time series, Astron. J. 112 (1996) 1709-1729.

[16] C. Torrence, G.P. Compo, A practical guide to wavelet analysis, Bull. Amer. Met. Soc. 79 (1998) 61-78. 